\title{
Assessment of Bone Health Education in US Multiple Myeloma and Solid Tumor Patients at Risk for Skeletal-Related Events
}

\author{
Darcy R Flora' \\ Katherine B Carlson ${ }^{2}$ \\ David C Fuehrer' \\ Benoit Cadieux ${ }^{3}$ \\ Guy Boike 4 \\ Jennifer Schenfeld ${ }^{2}$ \\ Kimberly A Lowe 2 \\ 'GRYT Health Inc., Rochester, NY, USA; \\ ${ }^{2}$ Center for Observational Research, \\ Amgen Inc., Thousand Oaks, CA, USA; \\ ${ }^{3}$ Global Medical Organization, Amgen \\ Inc., Thousand Oaks, CA, USA; \\ ${ }^{4}$ Karmanos Cancer Institute, Detroit, \\ MI, USA
}

\begin{abstract}
Purpose: Cancer patients with bone metastasis (BM) from solid tumors or multiple myeloma (MM) have an increased risk of painful skeletal-related events (SREs), which can decrease quality of life and increase mortality. Bone targeting agents (BTAs) can help delay or prevent SREs; however, a significant portion of eligible patients are not receiving BTA therapy. This study was conducted to understand patient awareness of cancer-related bone health and to identify opportunities to improve bone health education in cancer patients at risk of SREs.
\end{abstract}

Methods: The online BonE heAlth eduCatiOn Needs assessment (BEACON) survey included questions about patient demographics, cancer diagnosis and treatments (including BTA usage), and extent and satisfaction with bone health education received. Direct-topatient outreach was used to recruit patients. Eligible patients were US adults with a diagnosis of self-reported MM or BM from a solid tumor (breast, lung, or prostate cancer) within the past three years.

Results: Of 125 patients, $71 \%$ were diagnosed with solid tumors with BM and $29 \%$ with MM. At least one prior SRE was experienced by $57 \%$ of patients (38\% radiation to bone, $32 \%$ bone fracture, $22 \%$ spinal cord compression, and $19 \%$ surgery to bone), and $74 \%$ were currently receiving BTA therapy. Awareness of cancer bone health, protection strategies, and screening tests was low to moderate; patients were least informed of the impact of lifestyle changes $(38 \%)$ and specific cancer treatments $(\leq 35 \%)$ on bone health. Sixty-two percent of patients were not completely satisfied with the bone health education received. Patients generally wanted more information $(58 \%)$ and to receive information by more than one mode of communication.

Conclusion: Notable gaps in bone health education were observed in cancer patients at risk for SREs indicating an important need for improved communication and education strategies to promote better health outcomes.

Keywords: bone metastasis, bone targeting agents, fracture, radiation to bone, surgery to bone, spinal cord compression

\section{Introduction}

Breast, lung, and prostate cancer are the most commonly diagnosed cancers in the United States and are expected to account for $39 \%$ of new cancer cases and $35 \%$ of cancer deaths in 2020. ${ }^{1}$ Bone is a frequent site of metastasis for patients with advanced disease. Up to $89 \%$ of advanced prostate cancer, $75 \%$ of advanced breast cancer, and $40 \%$ of advanced lung cancer patients develop bone metastasis (BM) over the course of their disease. ${ }^{2-5}$ Multiple myeloma (MM), a cancer of the plasma
Correspondence: Darcy R Flora GRYT Health Inc., 919 Winton Road South, Suite 307, Rochester, NY, I46I8, USA

Tel + I 8444874798

Fax +15854863102

Email darcy@grythealth.com 
cells, is predicted to account for $2 \%$ of new cancer cases and deaths in the United States in $2020 .{ }^{1}$ It is the cancer type that most frequently affects the skeleton, with $90 \%$ of patients developing bone lesions. ${ }^{6}$ In contrast to lesions caused by BM in solid tumors, which may be osteolytic, osteoblastic, or of mixed type, bone lesions in MM patients are purely osteolytic. ${ }^{6,7}$ Irrespective of whether the mechanism is osteolytic and/or osteoblastic, the resultant dysregulation of normal bone remodeling that arises from cancer involvement in bone weakens the structural integrity of bone and increases the risk for painful and irreversible bone complications. ${ }^{5,6}$

Bone complications, referred to as skeletal-related events (SREs), include pathological fractures, spinal cord compression, radiation to bone (for severe pain), and surgery to bone (for stabilization). Rates of SREs, either at diagnosis or over the course of patient follow-up, are reported to be as high as $41 \%, 63 \%, 59 \%$, and $52 \%$ in patients with $\mathrm{MM}$ and bone metastatic breast, lung, and prostate cancer, respectively. ${ }^{8-10}$ Advanced cancer trial data suggest that these patients experience an SRE every three to six months. ${ }^{11}$ SREs are associated with decreased quality of life (QoL) and increased morbidity and mortality and, therefore, present a significant health and economic burden. ${ }^{8,12-14}$ After experiencing an initial SRE, patients report a clinically significant decline in QoL parameters including physical, functional, and emotional wellbeing. $^{15-17}$

Bone targeting agents (BTAs), such as bisphosphonates and the receptor activator of nuclear factor-kappa $\beta$ ligand (RANKL) inhibitor denosumab, have been shown to delay the progression of bone pain and reduce the incidence of SREs, thereby improving QoL. ${ }^{8,18}$ Cancer treatment guidelines recommend treatment with BTAs in patients at risk for SREs; however, real-world studies suggest that a large proportion of eligible patients do not receive BTAs. ${ }^{19,20}$ In fact, more than half of BTA-treated patients may not initiate BTA therapy until after experiencing an initial SRE. ${ }^{20}$ The reasons for low BTA usage are not well understood but may be related to supportive cancer care being delayed or overlooked.

Previous survey studies have focused on physician opinions and BTA utilization patterns; however, no prior studies leveraged patient opinions and/or experiences for assessment of bone health education and knowledge. ${ }^{21}$ To better understand bone health education practices in the patient community at risk for SREs, this study surveyed patients diagnosed with either solid tumors with BM or
MM. The survey was designed to understand: 1) the source, type, and extent of bone health education patients receive as part of supportive care, and 2) potential opportunities for improvement in cancer-related bone health education to enhance appropriate use of BTAs and health outcomes.

\section{Materials and Methods Survey Design}

A cross-sectional online survey study was conducted to evaluate the awareness of cancer-related bone health and SRE treatment options in cancer patients at high risk for SREs. The BonE heAlth eduCatiOn Needs assessment (BEACON) survey consisted of questions related to patient demographics, cancer diagnosis and treatments, experience with cancer-related SREs, knowledge of general cancer-related bone health, bone health protection, and screening for bone health, extent and overall satisfaction with bone health education received (amount, source, and mode), and use of a BTA. Most questions included predefined categorical options. Two open-ended questions were asked at the end of the survey to gain qualitative data insights and give patients the opportunity to elaborate on their experience with bone health education and management.

To assess knowledge around cancer-related bone health, patients were presented with six general bone health awareness statements and asked to identify which, if any, had been shared with them by a health care provider (HCP) since their diagnosis of $\mathrm{BM}$ or MM: 1) Bones are more fragile in individuals who have cancer, 2) Bones are more fragile in individuals who have received radiation therapy, 3) Bones are more fragile in individuals who have received chemotherapy, 4) Individuals with cancer have a greater risk of experiencing broken bones because of their cancer, 5) There are lifestyle changes individuals can make to help prevent broken bones caused by cancer, and 6) There are treatments available that help prevent broken bones caused by cancer. Patients could also indicate that none of the statements had been shared or that general bone health was not discussed with their $\mathrm{HCP}(\mathrm{s})$.

Patients were also asked to identify which, if any, bone health protection strategies and screening tests had been shared or recommended to them by an HCP. The protection strategies included: 1) use of calcium and/or vitamin D supplements, 2) regular physical activity and 
weight-bearing exercises, 3) lifestyle changes, and 4) treatment with BTAs. Screening tests included: 1) screening for osteoporosis or evaluation of bone mineral density, 2) checking blood calcium and/or vitamin D levels, and 3) assessing fracture risk. Again, patients could also indicate lack of awareness or recall of protection strategies or screening tests.

Development of the survey included pilot interviews with patients to ensure the survey functioned properly and that the questions were easily understood. The online survey was designed to take approximately 30 minutes to complete. Responses to all questions were required to minimize missing data. A HIPAA-compliant online platform was used to conduct the survey. The study was approved by Western Institutional Review Board (WIRB), an independent ethical review board (WIRB Study Number 1258908), and conducted in accordance with the Declaration of Helsinki.

\section{Study Recruitment and Population}

GRYT Health, a humanity-focused digital oncology company that empowers the cancer community to be in charge of their own health through education, engagement, and support, used a unique direct-to-patient approach for study recruitment. In addition to engaging members of the GRYT Health Cancer Community, patients were also engaged through established partnerships with cancer nonprofit organizations and support groups, patient-targeted outreach via social media platforms, paid targeted online advertising, and GRYT Health's annual Global Virtual Cancer Conference. Interested patients were screened, and eligible patients were sent a link to the online survey to be completed at their convenience. GRYT Health managed all patient interactions.

Eligible patients were at least 18 years of age, resided in the United States, and were diagnosed with selfreported MM or bone metastasis from breast cancer, lung cancer, or prostate cancer within three years of study recruitment. These types of solid tumors were included because of the high incidence of these cancer types in the United States and because they represent the majority of bone metastatic solid tumor patients in the United States initiating BTA therapy. ${ }^{1,22}$ Both SRE naïve patients and patients who had experienced an SRE prior to study participation were included. All patients provided electronic informed consent prior to participation in the BEACON survey and received compensation following survey completion.

\section{Data Analysis}

All analyses are descriptive, and the results are presented as number and percentage. For data analysis, patients were categorized as having a solid tumor (including breast, lung, and prostate cancer) or MM. Incomplete surveys were excluded from the data analysis.

\section{Results}

A summary of patient characteristics is presented in Table 1. A total of 125 cancer patients at risk for SREs completed the BEACON survey, including $71 \%(\mathrm{n}=89)$ with metastatic solid tumors and $29 \%(\mathrm{n}=36)$ with MM. Within the solid tumor group, $83 \%(\mathrm{n}=74)$ had breast cancer, $9 \%(\mathrm{n}=8)$ had prostate cancer, and $8 \%(\mathrm{n}=7)$ had lung cancer. The majority of all patients were female (79\%) with a mean age of 48 years; ages ranged from 26 to 79 years. Young adult cancer patients, or patients under the age of 40 , represented $34 \%$ of all patients. The geographical distribution of all patients was comparable to the population distribution among the four US census regions. Patients were generally well educated with $67 \%$ having completed at least a college degree, and nearly all (98\%) reported some type of medical insurance coverage. Fiftytwo percent of solid tumor patients were diagnosed with $\mathrm{BM}$ at the same time as their initial cancer diagnosis, and the remaining experienced $\mathrm{BM}$ after an initial cancer diagnosis (data not shown).

There was variability in the frequency of the type of SRE experienced prior to participation among solid tumor and MM patients (Figure 1). The most common type of SRE was radiation to the bone for solid tumor patients (40\%) and bone fracture for MM patients (47\%). Of all patients who had experienced an SRE, 25\% reported only one SRE, $21 \%$ reported two SREs, $15 \%$ reported three SREs, $8 \%$ reported four SREs, and 30\% reported five or more SREs. MM patients who had experienced an SRE were more likely to experience five or more SREs compared to solid tumor patients who had experienced an SRE (38\% vs. $26 \%$ ). Forty-three percent of all patients were SRE naïve; this percentage reflects study design and is not reflective of real-world prevalence of SREs. Seventy-four percent of all patients reported that they were actively receiving a BTA; usage of either the RANKL inhibitor denosumab or the bisphosphonate zoledronic acid was reported nearly exclusively and in nearly equal numbers (data not shown). Of the patients not actively receiving a BTA, 55\% indicated that they had experienced at least 
Table I Patient Characteristics

\begin{tabular}{|c|c|c|c|}
\hline Characteristics & All Patients, $n=125$ & Solid Tumor ${ }^{\mathrm{a}}, \mathrm{n}=\mathbf{8 9}$ & Multiple Myeloma, $n=36$ \\
\hline \multicolumn{4}{|l|}{ Sex, n (\%) } \\
\hline Female & $99(79)$ & $80(90)$ & $19(53)$ \\
\hline Male & $26(2 I)$ & $9(10)$ & $17(47)$ \\
\hline \multicolumn{4}{|l|}{ Age (years) } \\
\hline Range & $26-79$ & $26-79$ & $32-77$ \\
\hline Mean \pm standard deviation & $48 \pm 13$ & $45 \pm 12$ & $55 \pm 13$ \\
\hline \multicolumn{4}{|l|}{ US region, n (\%) } \\
\hline Northeast & $19(15)$ & $16(18)$ & $3(8)$ \\
\hline Midwest & $26(2 I)$ & $17(19)$ & $9(25)$ \\
\hline South & $45(36)$ & $33(37)$ & $12(33)$ \\
\hline West & $35(28)$ & $23(26)$ & $12(33)$ \\
\hline \multicolumn{4}{|l|}{ Highest level of education, n (\%) } \\
\hline Less than high school & $I(I)$ & I (I) & $0(0)$ \\
\hline High school or GED & $9(7)$ & $6(7)$ & $3(8)$ \\
\hline Some college or post-high school education/training & $31(25)$ & $19(21)$ & $12(33)$ \\
\hline College degree & $46(37)$ & $36(40)$ & $10(28)$ \\
\hline Graduate degree (Masters, Professional, or Doctorate) & $38(30)$ & $27(30)$ & II (3I) \\
\hline \multicolumn{4}{|l|}{ Medical insurance ${ }^{\mathrm{b}}, \mathrm{n}(\%)$} \\
\hline Private & $82(66)$ & $60(67)$ & $22(61)$ \\
\hline Medicaid & $23(18)$ & $18(20)$ & $5(14)$ \\
\hline Medicare & $20(16)$ & $12(13)$ & $8(22)$ \\
\hline Other: TRICARE, Veterans Affairs & $2(2)$ & I (I) & I (3) \\
\hline None & $2(2)$ & I (I) & I (3) \\
\hline \multicolumn{4}{|l|}{ Time since diagnosis, $\mathrm{n}(\%)$} \\
\hline Less than I year ago & $20(16)$ & $12(13)$ & $8(22)$ \\
\hline I year ago & $24(19)$ & $16(18)$ & $8(22)$ \\
\hline 2 years ago & $43(34)$ & 31 (35) & $12(33)$ \\
\hline 3 years ago & $38(30)$ & $30(34)$ & $8(22)$ \\
\hline
\end{tabular}

Notes: ${ }^{a}$ Includes breast $(n=74)$, prostate $(n=8)$, and lung $(n=7)$ cancer patients. ${ }^{b}$ Patients could select more than one response.

one SRE and, of those who had experienced an SRE, 39\% had experienced five or more SREs. In comparison, 58\% of patients actively taking a BTA had experienced an SRE and, of those who had experienced an SRE, 26\% had experienced five or more SREs.

Patients reported on the HCPs that provided cancerrelated bone health information (data not shown). Nearly all patients reported seeing an oncologist and 94\% received at least some bone health information from their oncologist, however, 33\% wished they had received more information from their oncologist and 4\% received no information from their oncologist. Nurse practitioners $(53 \%)$ and registered nurses (52\%) were also cited as common sources of bone health information. Less than $25 \%$ of patients reported receiving bone health information from each of the following HCPs: physical therapists, dieticians/nutritionists, pharmacists, patient navigators, and social workers.

Table 2 summarizes how patients responded to each general bone health awareness statement. Patients most commonly had awareness around the effect of cancer on the bone (64-68\%) and the availability of treatments to protect bone health (66\%). In patients not on BTA therapy, general bone health knowledge was low with no more than $45 \%$ for any of the statements; however, knowledge of the effect of cancer on the bone (45\%) and the availability of treatments to protect bone health (39\%) were noticeably lower compared to patients actively receiving a BTA (71-76\% and 75\%, respectively). Forty-one percent of patients were aware of more than three of the six general bone health awareness statements. Of the patients without knowledge of any general awareness statements, 12 of the $13 \mathrm{had}$ a solid tumor with BM diagnosis. 


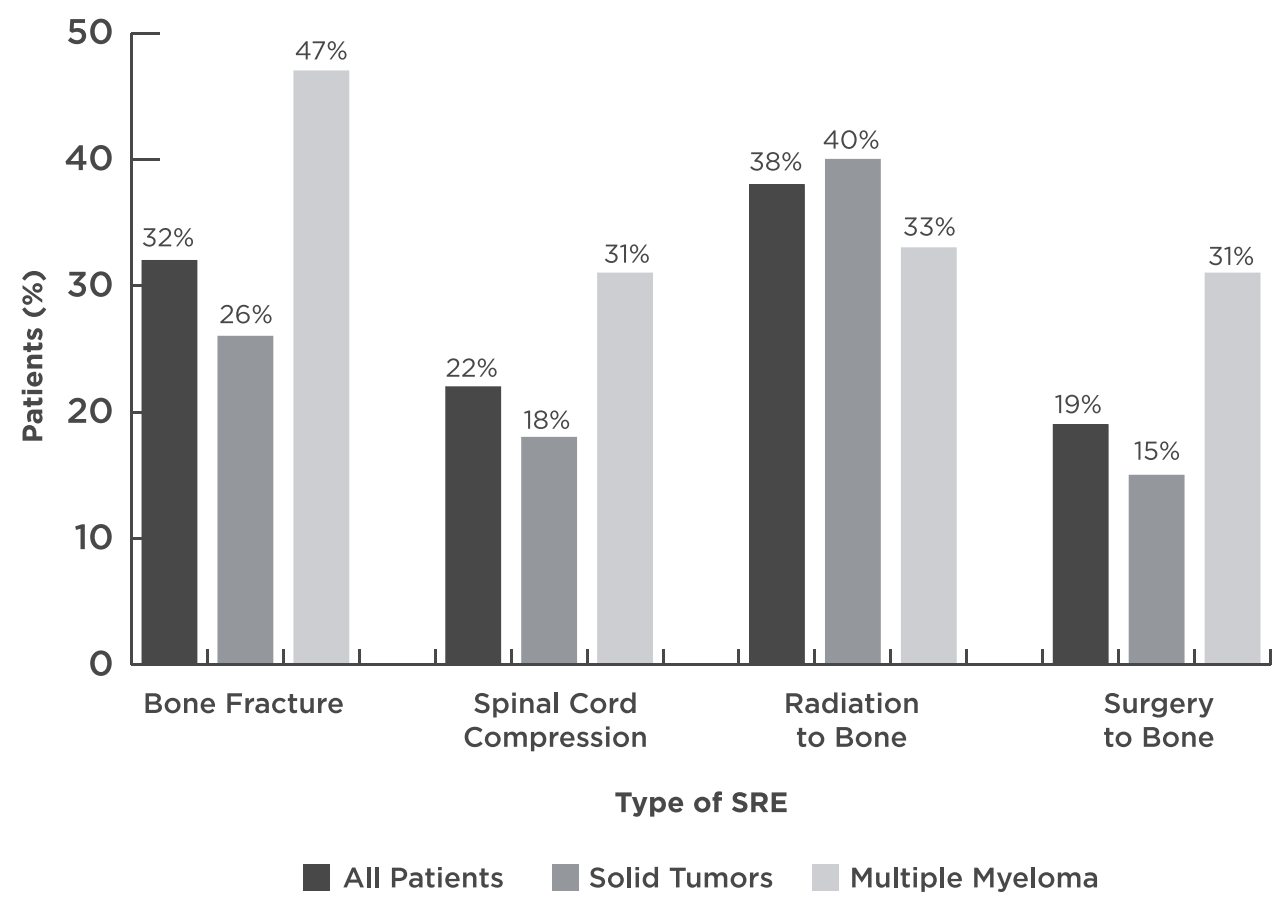

Figure I Type of skeletal-related event (SRE) reported by patients. Note: $43 \%(n=54$ of 125$)$ patients were SRE naive prior to survey participation.

Knowledge around bone health protection strategies and recommended screening is also summarized in Table 2. The majority (97\%) of patients were aware of at least one protection strategy. Patients were most commonly aware of the use of supplements (70\%) and BTAs (68\%) to protect bone health, but much less aware of the role of lifestyle changes $(22 \%)$ in protecting bone health. Thirtyeight percent of all patients had knowledge of at least three of the four protection strategies. At least one screening test was recommended to $78 \%$ of the patients. Only $8 \%$ of all patients were recommended an assessment for fracture risk. None of the three screening tests stated in the survey were recommended to $30 \%$ of the patients not actively on a BTA compared to $18 \%$ of patients on a BTA.

Amount and satisfaction with bone health information received is presented in Figure 2. More than half (58\%) of patients reported that they had received either not enough or no bone health information. It was more common for solid tumor patients not to receive any bone health information than $\mathrm{MM}$ patients (11\% vs. $3 \%$, respectively). Receiving too much information was rare (1\%). Thirtyeight percent of all patients were very or extremely satisfied with the amount of information received. Patients receiving a BTA $(41 \%)$ were more likely to be very or extremely satisfied with the amount of bone health information received compared to patients not on a BTA $(30 \%)$.
Bone health information was commonly received by patients during discussions with HCPs (88\%), which was also how patients preferred to receive the information (88\%) (Figure 3).

\section{Discussion}

Maintaining bone health in cancer patients is important to delay or prevent painful SREs which lead to decreases in QoL and are associated with an increased mortality. Previous studies have focused on prevalence of SREs, impacts of SREs to patients, and utilization of BTAs by examining health record or claims data and surveying physicians, with minimal engagement or understanding of patient awareness of cancer-related bone health. ${ }^{20,21}$ This study examined patient awareness of bone health to better understand the gaps in patient bone health education. Rather than surveying physicians to learn when, how, and what they inform their patients about bone health, this study recruited patients directly to learn about their experience and assess their knowledge of bone health, which offers an important perspective since patients are the experts on the patient experience. Patient recruitment was accomplished primarily through the GRYT Health Cancer Community and its relationships within the cancer community, which eliminated potential bias from the patient's $\mathrm{HCP}$ and/or treatment center. 
Table 2 Bone Health Information Shared or Recommendations Made by HCPs

\begin{tabular}{|c|c|c|c|}
\hline Bone Health Statement & $\begin{array}{l}\text { All Patients, } \\
\mathrm{n}=125\end{array}$ & $\begin{array}{l}\text { Solid Tumor } \\
\mathrm{n}=\mathbf{8 9}\end{array}$ & $\begin{array}{l}\text { Multiple Myeloma } \\
\mathrm{n}=\mathbf{3 6}\end{array}$ \\
\hline \multicolumn{4}{|l|}{ General bone health, $\mathrm{n}(\%)$} \\
\hline Bones are more fragile in individuals who have cancer & $85(68)$ & $56(63)$ & $29(8 \mathrm{I})$ \\
\hline Bones are more fragile in individuals who have received radiation therapy & $44(35)$ & $35(39)$ & $9(25)$ \\
\hline Bones are more fragile in individuals who have received chemotherapy & $34(27)$ & $21(24)$ & $13(36)$ \\
\hline $\begin{array}{l}\text { Individuals with cancer have a greater risk of experiencing broken bones } \\
\text { because of their cancer }\end{array}$ & $80(64)$ & $54(61)$ & $26(72)$ \\
\hline $\begin{array}{l}\text { There are lifestyle changes individuals can make to help prevent broken } \\
\text { bones caused by cancer }\end{array}$ & $48(38)$ & $30(34)$ & $18(50)$ \\
\hline There are treatments available to help prevent broken bones caused by cancer & $82(66)$ & $59(66)$ & $23(64)$ \\
\hline None of the above; HCPs have not discussed bone health & $13(10)$ & $12(13)$ & I (3) \\
\hline \multicolumn{4}{|l|}{ Bone health protection strategies, $\mathrm{n}(\%)$} \\
\hline Use of calcium and/or vitamin D supplements & $88(70)$ & $66(74)$ & $22(61)$ \\
\hline Regular physical activity and weight-bearing exercises & $67(54)$ & $46(52)$ & $21(58)$ \\
\hline Lifestyle changes (eg, stopping smoking or reducing alcohol consumption) & $28(22)$ & $20(22)$ & $8(22)$ \\
\hline Treatment with bone targeting/bone health agents & $85(68)$ & $61(69)$ & $24(67)$ \\
\hline None of the above; HCPs have not discussed preventative strategies & $4(3)$ & $2(2)$ & $2(6)$ \\
\hline \multicolumn{4}{|l|}{ Bone health screening tests, $\mathrm{n}(\%)$} \\
\hline Screening test for osteoporosis or evaluation of bone mineral density & $44(35)$ & $29(33)$ & $15(42)$ \\
\hline Blood test to check calcium and/or vitamin D levels & $88(70)$ & $64(72)$ & $24(67)$ \\
\hline Fracture risk assessment & $10(8)$ & $8(9)$ & $2(6)$ \\
\hline Not applicable; HCPs have not recommended screening tests & $27(22)$ & $20(22)$ & $7(19)$ \\
\hline
\end{tabular}

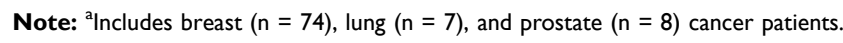
Abbreviation: $\mathrm{HCP}$, health care provider.

Study findings suggest that knowledge of general bone health and bone health protection strategies in patients at high risk for SREs was low to moderate. It is notable that $97 \%$ of patients in the study expressed awareness of at least one bone health protection strategy. Patients were most aware of the effects cancer had on the bone and that pharmacological agents were available to protect bones; however, for even these statements, one-third of patients indicated they were not informed of this information. The largest gaps in knowledge were around the effect of specific cancer treatments on the bone and lifestyle changes that can help protect bones. In open-ended responses, patients reported wanting to know: additional information about bone health supplements and BTAs, dietary recommendations, how to monitor bone health, safe activities to strengthen bones, statistics on SRE risk, and the science behind bone health and how their cancer specifically affected it. The majority of bone health information was conveyed during conversations with the patient's oncologist(s) and nurse(s). While it is important to continue bone health conversations with these HCPs, there is an opportunity for other HCPs to be more involved in providing bone health education to these patients as their knowledge is currently being underutilized. Additionally, conversations with HCPs are not enough; patients also want trustworthy information to be provided by additional modes of communication such as in printed or electronic materials (email, website, video, etc.).

Differences in the patients' SRE profile and/or bone health education experience were observed between specific patient groups within the study. Compared to solid tumor patients that had experienced an SRE, MM patients with a prior SRE on average experienced twice as many SREs and were more likely to experience five or more SREs. The type of SRE experienced also varied based on cancer type; MM patients most frequently reported bone fracture whereas solid tumor patients most frequently reported radiation to bone. Perhaps related to the significant relationship between MM and bone, study findings indicated that MM patients were slightly more aware of general cancer-related bone health compared to patients with BM from solid tumors and less likely to receive no bone health information from their HCP. The study also allowed for comparison of patients actively receiving or 
A

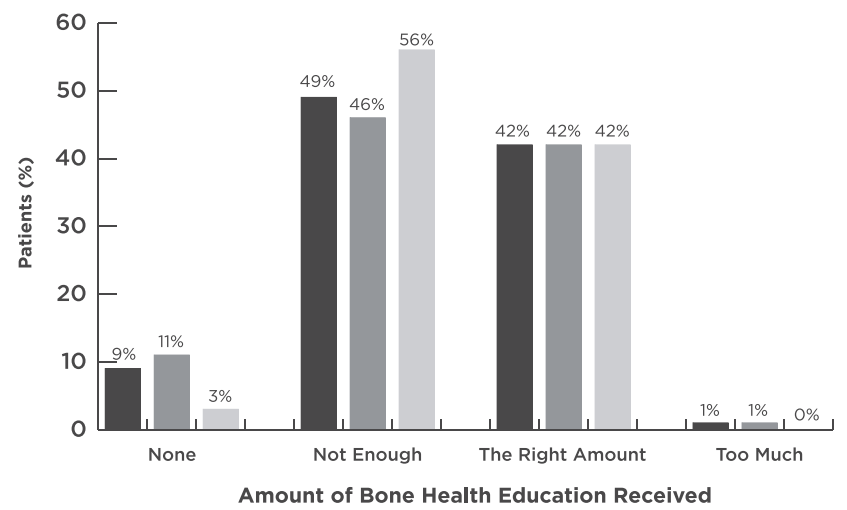

B

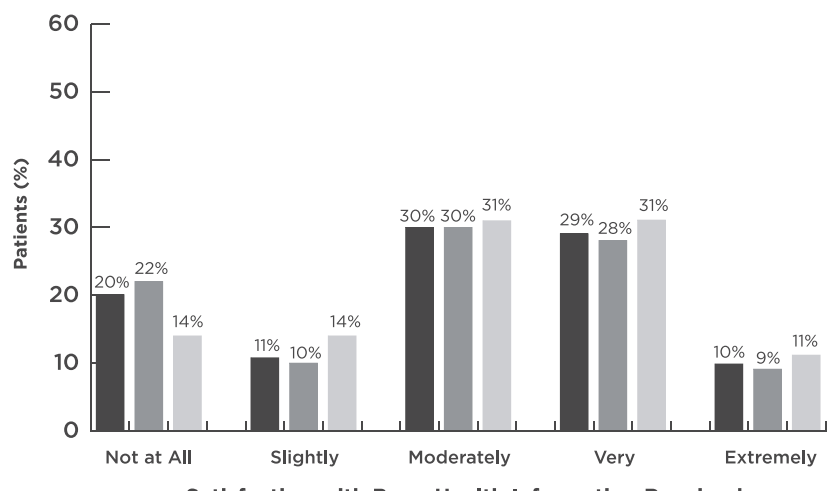

Satisfaction with Bone Health Information Received

All Patients Solid Tumors Multiple Myeloma

Figure 2 Amount and satisfaction of bone health information received by patients. (A) Amount of bone health information received. (B) Satisfaction with bone health information received.

A

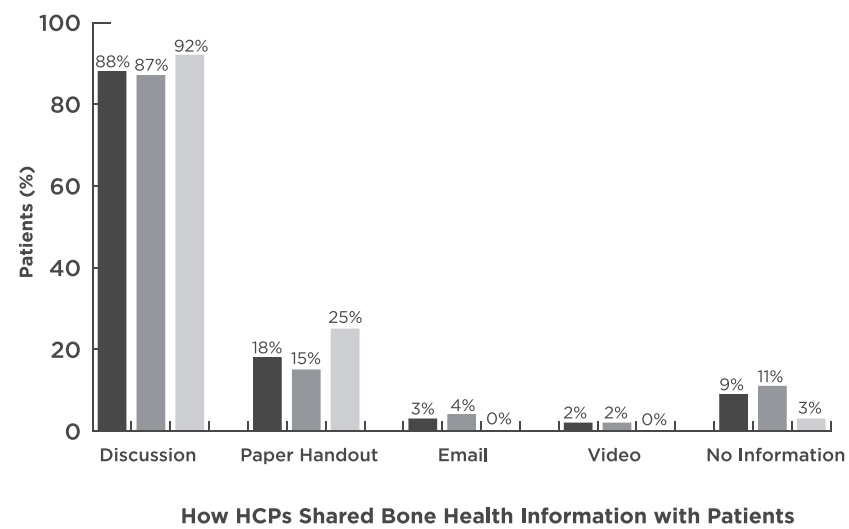

B

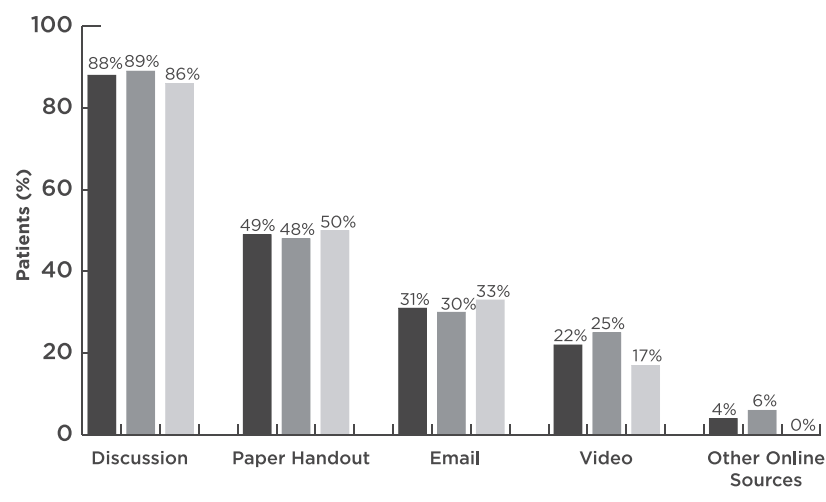

How Patients Want to Receive Bone Health Information

All Patients

Solid Tumors

Multiple Myeloma

Figure 3 Sharing of bone health information. (A) How HCPs shared bone health information with patients. (B) How patients want bone health information to be shared.

not receiving a BTA. Twenty-six percent of patients were not actively taking a BTA, although the reason for not currently taking a BTA is unknown. Possible explanations include a recent cancer diagnosis, perceived low risk of SREs, an interruption in BTA therapy, or short life expectancy. ${ }^{21,23}$ Additionally, it is unknown how many of these patients were BTA naïve and how many had previously received BTA therapy. Although a similar percentage of patients with a prior SRE were reported among patients actively receiving or not receiving a BTA, differences were noted in their level of bone health knowledge and satisfaction with bone health information received. Overall general bone health knowledge in patients not receiving a BTA was low, with no more than $45 \%$ of the patients aware of any of the six statements presented. Specifically, knowledge about the effect of cancer on bones and that treatments were available to help prevent broken bones was markedly reduced in the patients not receiving a BTA. This patient group also reported lower satisfaction with bone health education received. These observations within specific patient groups help to shed light on shortcomings in patient bone health awareness.

There are limitations that should be considered when interpreting the results from this study. The study consisted of mostly metastatic breast cancer and MM patients. Due to challenges in recruiting lung and prostate patients 
with BM, these patients only accounted for $12 \%$ of the study population and are therefore not generalizable to patients with these types of cancer. Possible reasons for these challenges include aggressiveness of the disease, competing priorities with advanced disease, stigma associated with the disease, and caregivers, rather than patients, being the better point of direct contact. Due to the short life expectancy of lung cancer patients, studies around the incidence of BM and SREs have been limited. ${ }^{12,18}$ Nevertheless, even small amounts of information can be informative. Additionally, this study may not be representative of the general cancer population. The digital recruitment method favors patients that were more internet savvy and that were seeking connection and/or information. As a result, patients in this study were notably younger, more educated, and likely more engaged and/ or informed about their general health. These characteristics suggest that the study population may be more informed and take more action to preserve their bone health compared to the average patient with a similar diagnosis. Despite these limitations, this study successfully utilized direct-to-patient recruitment methods to provide valuable insights into patient awareness of cancer-related bone health. Furthermore, patient feedback revealed that the survey unintentionally served as an educational tool to patients, particularly in instances where there was a deficiency in bone health education received.

\section{Conclusion}

Gaps in bone health education and awareness were observed in this study of MM and metastatic breast, lung, and prostate cancer patients at risk for SREs. This study provides evidence supporting the need for both patient- and provider-oriented interventions to prevent painful and irreversible SREs. While patients are primarily receiving bone health information from their oncologists and nurses, there is an opportunity for these HCPs to more effectively communicate this information and for other HCPs to become more involved in educating patients about bone health. In particular, patients lacked adequate information on lifestyle changes and physical activities that could be implemented to help preserve and protect bone health. As advancements in cancer therapies continue to extend life expectancy in advanced cancer patients, it will be important to place a stronger emphasis on early and effective supportive care, such as bone health education, for improved health outcomes. Future studies in oncologic care should attempt to gather information on patient's perspective of all aspects of the treatment process, including education regarding options, rationale, potential side effects, and prevention of side effects.

\section{Funding}

This study was funded by Amgen. Amgen contributed to study conception and design, data analysis and interpretation, and preparation of the manuscript.

\section{Disclosure}

Dr Darcy R Flora reports GRYT Health has received payment from Amgen to design, conduct, and analyze data from the study and to prepare the manuscript; GRYT Health also has received payment from Amgen for other research and for sponsorship of GRYT Health's Global Virtual Cancer Conference. Dr Katherine B Carlson is a former employee of Amgen and is a current employee of Moderna. Dr Benoit Cadieux, Ms Jennifer Schenfeld, and Dr Kimberly A Lowe are employees of and hold stock in Amgen. The authors report no other conflicts of interest in this work.

\section{References}

1. Siegel RL, Miller KD, Jemal A. Cancer statistics, 2020. CA Cancer J Clin. 2020;70(1):7-30. doi:10.3322/caac.21590

2. Huang JF, Shen J, Li X, et al. Incidence of patients with bone metastases at diagnosis of solid tumors in adults: a large population-based study. Ann Transl Med. 2020;8(7):482. doi:10. 21037/atm.2020.03.55

3. Hernandez RK, Wade SW, Reich A, Pirolli M, Liede A, Lyman GH. Incidence of bone metastases in patients with solid tumors: analysis of oncology electronic medical records in the United States. BMC Cancer. 2018;18(1):44. doi:10.1186/s12885-017-3922-0

4. Suva LJ, Washam C, Nicholas RW, Griffin RJ. Bone metastasis: mechanisms and therapeutic opportunities. Nat Rev Endocrinol. 2011;7(4):208-218. doi:10.1038/nrendo.2010.227

5. Coleman RE. Skeletal complications of malignancy. Cancer. 1997;80 (8 Suppl):1588-1594.

6. Marino S, Roodman GD. Multiple myeloma and bone: the fatal interaction. Cold Spring Harb Perspect Med. 2018;8(8):a031286. doi:10.1101/cshperspect.a031286

7. Roodman GD. Mechanisms of bone metastasis. $N$ Engl J Med. 2004;350(16):1655-1664. doi:10.1056/NEJMra030831

8. Berenson JR, Lichtenstein A, Porter L, et al. Efficacy of pamidronate in reducing skeletal events in patients with advanced multiple myeloma. Myeloma Aredia Study Group. $N$ Engl J Med. 1996;334 (8):488-493. doi:10.1056/NEJM199602223340802

9. Kim C, Bhatta S, Cyprien L, Fonseca R, Hernandez RK. Incidence of skeletal-related events among multiple myeloma patients in the United States at oncology clinics: observations from real-world data. J Bone Oncol. 2019;14:100215. doi:10.1016/j.jbo.2018.100215

10. Oster G, Lamerato L, Glass AG, et al. Natural history of skeletal-related events in patients with breast, lung, or prostate cancer and metastases to bone: a 15-year study in two large US health systems. Support Care Cancer. 2013;21(12):3279-3286. doi:10.10 07/s00520-013-1887-3 
11. Coleman RE. Clinical features of metastatic bone disease and risk of skeletal morbidity. Clin Cancer Res. 2006;12(20 Pt 2):6243s-6249s. doi:10.1158/1078-0432.CCR-06-0931

12. Cetin K, Christiansen CF, Jacobsen JB, Norgaard M, Sorensen HT. Bone metastasis, skeletal-related events, and mortality in lung cancer patients: a Danish population-based cohort study. Lung Cancer. 2014;86(2):247-254. doi:10.1016/j.lungcan.2014.08.022

13. So A, Chin J, Fleshner N, Saad F. Management of skeletal-related events in patients with advanced prostate cancer and bone metastases: incorporating new agents into clinical practice. Can Urol Assoc J. 2012;6(6):465-470. doi:10.5489/cuaj.12149

14. Norgaard M, Jensen AO, Jacobsen JB, Cetin K, Fryzek JP, Sorensen HT. Skeletal related events, bone metastasis and survival of prostate cancer: a population based cohort study in Denmark (1999 to 2007). J Urol. 2010;184(1):162-167. doi:10.1016/j.juro.2010.03.034

15. Weinfurt KP, Li Y, Castel LD, et al. The significance of skeletal-related events for the health-related quality of life of patients with metastatic prostate cancer. Ann Oncol. 2005;16(4):579-584. doi:10.1093/annonc/mdi122

16. Weinfurt KP, Castel LD, Li Y, Timbie JW, Glendenning GA, Schulman KA. Health-related quality of life among patients with breast cancer receiving zoledronic acid or pamidronate disodium for metastatic bone lesions. Med Care. 2004;42(2):164-175. doi:10. 1097/01.mlr.0000108746.69256.45

17. Kunikane H, Yokota I, Katakami N, et al. Prospective analysis of the association between skeletal-related events and quality of life in patients with advanced lung cancer (CSP-HOR13). Oncol Lett. 2019;17(1):1320-1326. doi:10.3892/ol.2018.9680
18. Brodowicz T, O’Byrne K, Manegold C. Bone matters in lung cancer. Ann Oncol. 2012;23(9):2215-2222. doi:10.1093/annonc/ mds009

19. McGrath LJ, Overman RA, Reams D, et al. Use of bone-modifying agents among breast cancer patients with bone metastasis: evidence from oncology practices in the US. Clin Epidemiol. 2018;10:1349-1358. doi:10.2147/CLEP.S175063

20. Hernandez RK, Adhia A, Wade SW, et al. Prevalence of bone metastases and bone-targeting agent use among solid tumor patients in the United States. Clin Epidemiol. 2015;7:335-345. doi:10.2147/ CLEP.S85496

21. Henry D, von Moos R, Body JJ, et al. Bone-targeted agent treatment patterns and the impact of bone metastases on patients with advanced breast cancer in the United States. Curr Med Res Opin. 2019;35 (3):375-381. doi:10.1080/03007995.2018.1558849

22. Qian Y, Bhowmik D, Kachru N, Hernandez RK. Longitudinal patterns of bone-targeted agent use among patients with solid tumors and bone metastases in the United States. Support Care Cancer. 2017;25(6):1845-1851. doi:10.1007/s00520-017-35 83-1

23. Butler AM, Cetin K, Hernandez RK, et al. Treatment dynamics of bone-targeting agents among men with bone metastases from prostate cancer in the United States. Pharmacoepidemiol Drug Saf. 2018;27 (2):229-238. doi: $10.1002 /$ pds.4360

\section{Publish your work in this journal}

Cancer Management and Research is an international, peer-reviewed open access journal focusing on cancer research and the optimal use of preventative and integrated treatment interventions to achieve improved outcomes, enhanced survival and quality of life for the cancer patient.
The manuscript management system is completely online and includes a very quick and fair peer-review system, which is all easy to use. Visit http://www.dovepress.com/testimonials.php to read real quotes from published authors. 